\title{
Photodynamic antimicrobial chemotherapy and ultraconservative caries removal linked for management of deep caries lesions
}

\author{
M.A.S. Melo a , J.P.M.L. Rolim ${ }^{b}$, V.F. Passos ${ }^{c}$, R.A. Lima ${ }^{b}$, I.C.J. Zanin ${ }^{\text {d }}$, Bruna M. Codes ${ }^{b}$, \\ S.S. Rocha ${ }^{\mathrm{b}}$, L.K.A. Rodrigues ${ }^{\mathrm{b}, *}$ \\ a University of Maryland School of Dentistry, Department of Endodontics, Prosthodontics and Operative Dentistry, Baltimore, MD, USA \\ ${ }^{\mathrm{b}}$ Federal University of Ceará, Post graduate Program in Dentistry, Faculty of Pharmacy, Dentistry and Nursing, Fortaleza, Ceará, Brazil \\ c University of Fortaleza, School of Dentistry, Fortaleza, Ceará, Brazil \\ d Federal University of Ceará, Faculty of Dentistry, Sobral, Brazil
}

\section{A R T I C L E I N F O}

\section{Article history:}

Received 12 June 2015

Received in revised form 17 September 2015

Accepted 24 September 2015

Available online 30 September 2015

\section{Keywords:}

Dental caries

LED

Photodynamic antimicrobial therapy

Clinical trial

\begin{abstract}
A B S T R A C T
Background: Ultraconservative removal of carious tissue is becoming increasingly highlighted for management of deep caries lesions, and combined with an antimicrobial photochemistry-based treatment modality (PACT), this approach can be enhanced favoring dental tissue repair and preservation. The aim was to investigate the effectiveness of PACT using a light emitting diode (LED) associated with a photosensitizer toluidine blue ortho (TBO) on deep caries lesions.

Methods: For that, a single blind, randomized, controlled, split-mouth clinical trial where 45 patients with at least two deep carious lesions on permanent posterior teeth was performed. The primary intervention was deep caries lesion management with disinfection of remaining dentin tissue using PACT. Bacterial counts were measured following treatments as the main outcome. The remaining dentinal samples of each lesion were treated with either non-PACT-control or PACT. The PACT procedure were characterized by $100 \mu \mathrm{g} \mathrm{mL}^{-1} \mathrm{TBO}$ followed by $94 \mathrm{~J} \mathrm{~cm}^{-2}$ LED irradiation. Samples of dentin were collected before and immediately after treatments for microbiological analysis of total viable bacteria, mutans streptococci and Lactobacillus spp. counts. Microbial reduction was data were submitted to unpaired $t$ test $(\alpha=5 \%)$. Results: PACT led to statistically significant reductions in mutans streptococci ( $1.08 \pm 1.20$ log), Lactobacillus spp. $(1.69 \pm 1.37 \mathrm{log})$, and total viable bacteria $(1.07 \pm 1.01 \mathrm{log})$ compared to the control, which showed $\log$ reductions respectively of $0.05 \pm 0.49,0.52 \pm 0.89$, and $0.47 \pm 0.77$ for the same microorganisms. Conclusion: Dentin from deep carious lesions treated with PACT showed a decrease in cariogenic microbial load.
\end{abstract}

(C) 2015 Elsevier B.V. All rights reserved.

\section{Introduction}

Deep caries management is a challenging approach in everyday dental practice due to the imminent risk of pulp exposure during the caries excavation procedures [1]. When treating deep caries lesions, the majority of failures were found to be in association with the pulp and bacterial infection, eventually leading to endodontic treatment or removal of the tooth [2]. The removal of all infected and affected dentin during complete excavation is the traditional treatment of choice for patients with caries lesion in advanced stages into dentin before placing a restoration [3]. This approach advocates the tissue removal as a prevention of further

\footnotetext{
* Corresponding author at: Post graduate Program in Dentistry, Faculty of Pharmacy, Dentistry and Nursing, Federal University of Ceará, 944Cap Francisco Pedro Street-Rodolfo Teófilo, Zip Code: 60430 170, Brazil. Fax: +55 8533668232.

E-mail address: lidianykarla@yahoo.com (L.K.A. Rodrigues).
}

cariogenic activity due to remaining bacteria in the demineralized inner layer. However, the unnecessary removal of dentin tissue and the possible exposure of the pulp are predictable, particularly in young patients [4].

Minimally invasive dentistry has emphasized the maximum tissue preservation in the management of deep dentin caries lesions. Conservative or ultraconservative removal of carious tissue has been proposed as a promising approach to promote the preservation of inner layer reaching bacterial reduction after a certain period of time and also leading to a possibility of the remineralization occur in the affected dentin $[5,6]$. Considering the microbiological point of view, photodynamic antimicrobial chemotherapy (PACT) may be a potential complementary treatment for permanent molars with deep carious lesions in a one-visit procedure since its effect is immediate [7]. This therapy involves photosensitizing agents, such as toluidine blue ortho (TBO), which are activated by irradiation with light of a specific wavelength to generate 
cytotoxic species, including singlet oxygen and free radicals capable of damaging essential components of the cells or modifying metabolic activities in an irreversible way, resulting in a faster bacterial killing effect $[8,9]$.

Previous studies have shown that PACT is able to reduce cariogenic bacteria, especially Streptococcus mutans, in planktonic culture [10-12]. Others studies have used dentin substrate and different caries models to reproduce clinical conditions and favorable results with regard to PACT were obtained [13-15]. These results in association with the technique benefits such as rapid noninvasive topical application of the photosensitizer in the carious lesion, the bacterial killing after a short exposure to light and the unlikely chance for resistance development have suggested a potential clinical applicability of PACT.

However, there exists a gap between what can be achieved through a clinical intervention and what is accomplished in pre-clinical studies. For instance, using the established light/photosensitizer parameters and the lack of outcomes for this therapy under real situation, factors including the presence of a mixed bacterial community enclosed within the demineralized dentin structure reinforces the need for a clinical investigation of PACT as a complementary therapy in caries management. Therefore, we designed a randomized, controlled trial (RCT) to analyze the effectiveness of this approach. In the current study, we tested the hypothesis that PACT will significantly reduce bacterial viability in remaining dentin.

\section{Materials and methods}

\subsection{Trial design}

This was a single blind (patient), randomized, controlled, intraindividual comparation (split-mouth), 2-group (intervention and control), clinical trial conducted between July 2010 and July 2012 in order to evaluate the antimicrobial effect of PACT in vivo. The study was approved by the institutional ethical review board and presented the clinical trial registration (Brazilian Registry of Clinical Trials U1111-1154-6303). All patients provided written informed consent to participate in the study. Research team consisted of a lead coordinator, an operative dentistry specialist to perform clinical procedures, a microbiologist to perform microbiological analysis, and a support team of general dentists.

\subsection{Patient's recruitment and inclusion}

Healthy male and female subjects older than 18 years-old who were referred for restorative treatment at Operative Dentistry Clinical Department, were recruited to participate in this study. Fig. $1 \mathrm{~A}$ shows the trial profile for this study. Eligible patients should meet the inclusion criteria: (1) moderate to deep dentin occlusal caries lesions in posterior teeth, corresponding to $\geq 2 / 3$ dentin, (2) one pair of bilateral caries lesions, of equal severity but not necessarily size, localized on upper or lower arch and (3) the presence of a well-defined radiolucent zone between the caries lesion and the pulp identified by bitewing radiographs.

The exclusion criteria of patients were as follows: (1) periapical radiograph showing pulp involvement, apical radiolucency, abscess or fistula associated (2) spontaneous pain, no response to cold pulp test; swelling in periodontal tissues, and abnormal tooth mobility, (3) use of antibiotics 3 months before or during the study and (4) patients that refused to sign the informed consent.

Four dentists were trained to identify eligible caries lesions by clinical examination and bitewing radiograph evaluation. However, only one professional conducted the processes of caries excavation and treatment application. After applying the inclusion and exclusion criteria, 50 patients (100 teeth) were selected.

\subsection{Sample size and randomization}

This trial followed a set of recommendations reported by the CONSORT 2010 Statement. According to a $t$-test for a two-treatment comparison which involves the use of pilot data [15], a minimum of 35 patients were needed to detect a $80 \%$ difference in bacterial reduction (i.e. $80 \%$ difference in PACT) between the control and the treated sides (Biostat 5.0, $\alpha=5 \%$ power test at 0.85) [16]. In this study 45 patients were recruited to compensate possible dropout during the experimental period.

A computer-generated randomization list by blocks of size 2 scheme (Excel) was used to randomize the sides of treatment application where each patient's mouth could contain a possible permutation of the two treatments in a random order. After this, one of the dentists (VP) manages the enrolled patients for allocation sequence. The blinding process for the operator was not possible to perform due to light activation related to PACT procedures. Patients were blinding by using dark laser safety glass during the procedures.

\subsection{Clinical procedures}

The operator had been trained and calibrated prior to this study for purposes of collecting dentin samples. Initially, the area of selected tooth was isolated with rubber dam followed by dental prophylaxis and initial cavity preparation using high-speed hand piece under copious air/water spray and diamond burs (\#FG 1019; KG Sorensen, Cotia, SP, Brazil). The central cariogenic biomasses was removed and complete excavation of carious tissue at the lateral walls of the cavity and at the enamel-dentin junction was performed using carbide round burs (\#4/6; KG Sorensen) at low speed. Subsequently, caries sampling from remaining dentin at pulp floor was managed using a sterile binangle discoid spoon. Two independent samples from this area were handled before and immediately after treatments for microbiological analysis.

\subsection{PACT protocol (photosensitizer and light source)}

After the baseline dentin collection, the remaining carious dentin was subjected to one of the treatments: $0.89 \% \mathrm{NaCl}$ solution (control group) or PACT (experimental group) (Fig. 1B). Briefly, for PACT procedures, toluidine blue ortho (TBO) (Sigma, St. Louis, MO, USA) was dissolved in deionized water at concentration of $100 \mu \mathrm{g} \mathrm{mL}^{-1}$ ). $10 \mu \mathrm{L}$ of TBO solution was applied in the selected area using pippete tip and 5 min-pre irradiation time was set up. A red light-emitting diode $(\lambda \sim 630 \mathrm{~nm}$; output: $150 \mathrm{~mW}$; MM Optics, São Carlos-SP, Brazil) connected to an optical fiber $(\varnothing=6 \mathrm{~mm})$ was used to deliver the total energy $94 \mathrm{~J} \mathrm{~cm}^{-2}$. Irradiation was performed at a distance of $2.0 \mathrm{~mm}$ from the cavity floor. The chosen parameters were based on the previous pre-clinical study (Lima et al. [29]). For the control group, the selected area received an equal volume of sterile $0.89 \% \mathrm{NaCl}$ solution instead of $\mathrm{TBO}$ and patients were submitted to a waiting time similar to that used for irradiation for blinding methodology purpose.

After the second sample was taken from an adjacent area, the lesion was washed with autoclaved distilled water for approx. $30 \mathrm{~s}$ until the photosensitizer was completely removed. A visual examination was performed to verify if TBO solution was completed washed out. All teeth were conventionally restored using the following materials: calcium hydroxide lining material (Dycal, Dentsply, Surrey, UK) and resin-based glass ionomer (Fuji II ${ }^{\circledR}, \mathrm{GC}$ America Inc., Alsip, IL, USA). A schematic representation of clinical and laboratorial procedures is presented in Fig. 1B. 
A
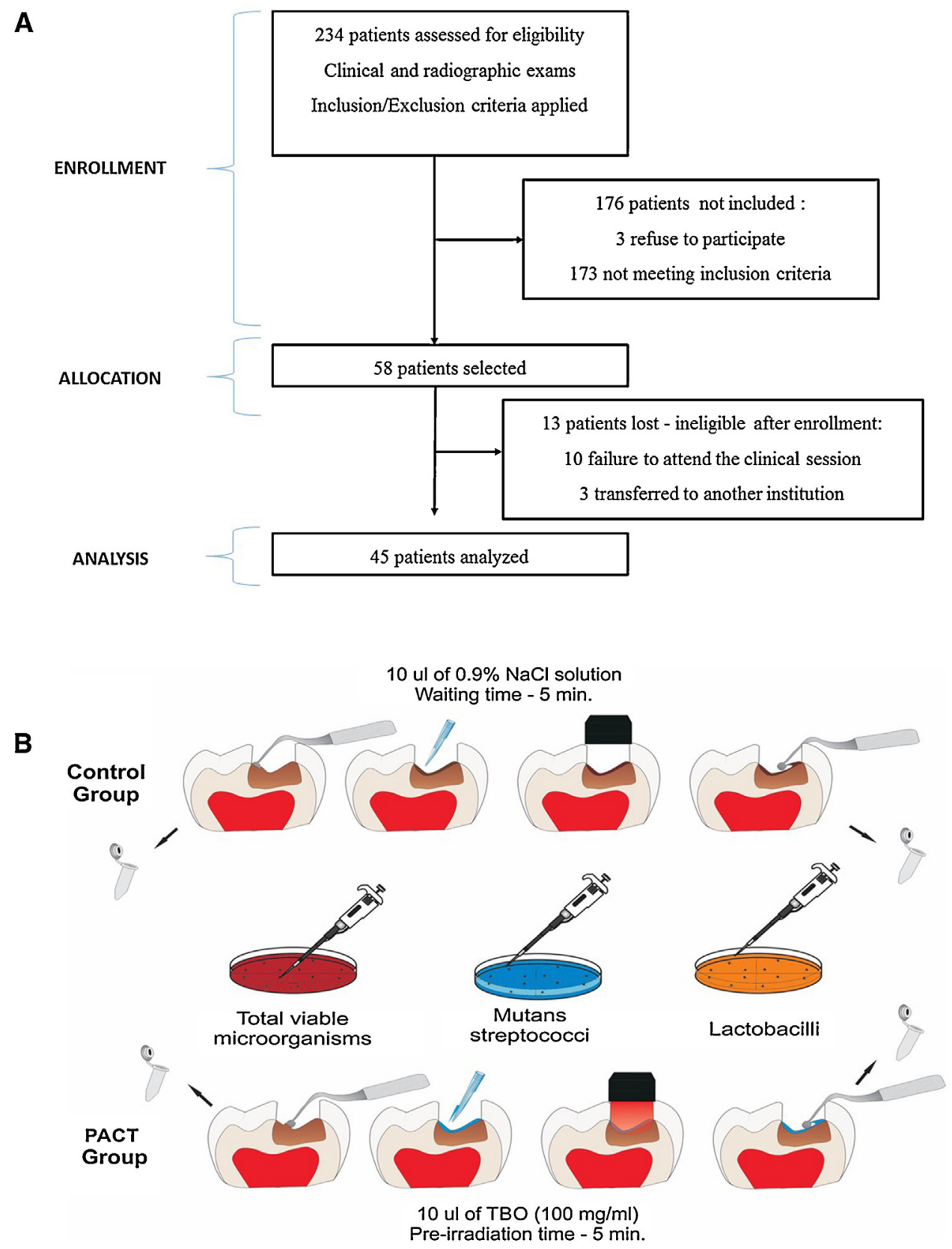

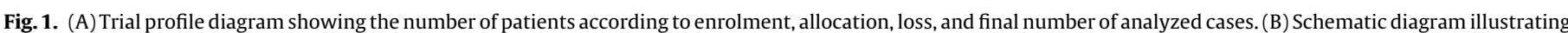

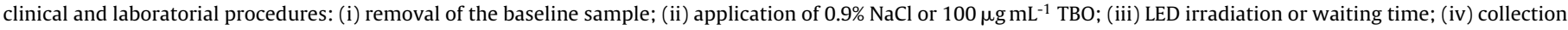
of post treatment sample; $(v)$ restoration of the tooth and microbiological analysis.

\subsection{Microbiological procedures}

Samples collected prior and after treatments were weighted using pre-weighed micro centrifuge tubes. Dentin weight was calculated by the difference between the weight of the set (tube + sample) and the previously determined weight of the tube without dentin. A $0.89 \% \mathrm{NaCl}$ solution $\left(1 \mathrm{~mL} \mathrm{mg}^{-1}\right)$ and glass beads were added to tubes following agitation for $1 \mathrm{~min}$ in a cell disruptor (Disrupter Genie, Precision Solutions, Rice Lake, WI, USA) in order to detach the bacterial cells. Afterwards, the suspension was serially diluted. The samples were plated in triplicate to assess microorganism viability, on the following culture media: blood agar to total microorganisms, Mitis Salivarius agar containing bacitracin and sucrose for mutans streptococci and rogosa agar for Lactobacilli counts. Agar plates were then incubated at $37^{\circ} \mathrm{C}, 5 \% \mathrm{CO}_{2}$ for $48 \mathrm{~h}$ before enumerating the viable microorganisms. The CFU counts were performed by a blinded examiner and expressed as colony-forming units (CFU) per milligram of carious dentin. The microbiological data were transformed into $\log _{10} \log _{10}$ reductions were used to show the relative number of live bacteria eliminated from a surface by the treatments. Log reduction (LR) was determined by calculating the difference between the $\log _{10}$ of the baseline measurement ( $\mathrm{CFU} / \mathrm{mg}$ of dentin) and the $\log _{10}$ of the measurement following each studied condition.

\subsection{Statistical analysis}

The normality distribution of the data and equality of variances were checked using the Kolmogorov-Smirnov and Levene tests, respectively. These data were analyzed using an unpaired $t$-test. The significance level was set at $5 \%$. The software SPSS was used. 


\section{Results}

We evaluated 234 patients for eligibility to participate in this study. Of these patients, 176 were excluded because they did not meet the inclusion criteria, refused to participate, or underwent treatment at other clinics. Thus, 58 patients had their teeth randomly allocated for PACT or control procedures (Fig. 1A); 13 were lost to not return to scheduled appointments. Forty-five patients were included in the analysis resulting in 90 lesions for the study. There were no changes to the outcome measures after the trial started. Patients were followed up for six months after the study and no patient reported any pain or sensitivity after the clinical procedures.

As shown in Fig. 2, the results showed that PACT significantly reduced viable counts of all tested microorganisms and the data are plotted for each evaluated medium. For total microorganism, the mean log bacterial counts recovered from the carious dentin before and after the treatments in the control group was 5.18 and 4.80 , respectively, demonstrating log reduction of 0.47 . PACT group showed values of 5.10 for baseline and 4.45 after the treatment reaching a log reduction of 1.07 ; which it was statistically different from control $(p=0.008)$.

After PACT, the greatest log reduction of 1.69 was achieved for Lactobacilli. The reached log reduction value for PACT treatment values was significantly different from log reduction value for control of $0.52(p=0.003)$. Similar significant difference was found between log reduction values of 1.08 after PACT treatment for mutans streptococci comparing with 0.50 in the respective group control $(p<0.0001)$.

\section{Discussion}

PACT is a unique treatment modality which a photosensitizer is activated by a light source with suitable wavelength to reach an antibacterial effect. Its application has been recently expanded to dentistry and it has received renewed attention in the context of dental caries management $[17,18]$. The non-invasive nature of PACT is aligned with the conservative approaches for caries removal and enhancement of pulp preservation, such as stepwise excavation, where the extension and amount of carious dentin removed during the procedures are reduced tending to preserve the amounts of demineralized dentin [19]. This cariesaffected demineralized dentin area is target point to PACT in caries management aiming to eliminate residual bacteria. Due its specific mechanism of action, PACT offers the particular advantage of immediate bacterial reduction; condition only reached by other approaches after the treatment stages of two-visit with long time interval between them (approx. three to six months) [5].

This randomized, clinical trial evaluated the use of PACT in a single visit in reducing bacterial counts in demineralized but still contaminated dentin during the management of deep caries lesions in adult's patients. In this intra-individual comparison, a single application of PACT was found to reduce significantly all analyzed bacterial group species: mutans streptococci, Lactobacilli and total microorganisms, which are suggested representative of dentin caries lesions [20]. The PACT achieved log reduction ranging from 1.07 to 1.69 . The prompt effect of this approach in bacterial population is believed to reflect the generation of reactive oxygen species (ROS), such as singlet oxygen and free radicals, which are cytotoxic to bacterial cells [21]. ROS can cause bacterial lethal injury by means of damage to an assorted range of target, mainly, bacterial DNA and the cytoplasmic membrane [22]. As trial limitations, the used sampling technique presents inherent variability due to microbiological procedures.
The relative high susceptibility of cariogenic bacteria, such as S. mutans and Lactobacillus acidophilus, to PACT using a diverse collection of photosensitizers and light sources is adequately documented [23-25]. Others have examined the effectiveness of PACT with TBO associated to a LED as light source and innumerous investigations have shown great bacterial reduction (log reduction up to 5) or even the fully elimination of, when these bacteria are suspended in planktonic cultures $[8,26]$ or organized in in vitro biofilms $[11,26,27]$. When PACT is tested under conditions more similar to those found in the mouth, i.e. by models using dentin samples under oral environment $[13,28]$, the bacterial reduction are minimized.

According to FDA- and ISO-established performance criteria, a minimum of a 3-log reduction must be achieved to state a clinically relevant pertinent antimicrobial efficacy (99.9\% percentage reduction). In our prior pilot study [30] elaborated to evaluate the processes for this main study and to assess the outcome of PACT-related control groups: only photosensitizer and only light irradiation, the log reduction values ranged from -0.12 to 2.68 , with the lowest value obtained for the control group and the highest for PACT group. Previous reports from our research group using similar protocol (LED; $94 \mathrm{~J} \mathrm{~cm}^{-2}$; TBO at $100 \mu \mathrm{g} \mathrm{mL}^{-1}$ ) were able to promote great bacterial $\log$ reduction $\left(>3 \log _{10}\right)$ in dentin caries produced by in vitro and in situ models [14,29]. However, the ample microbial reduction of $3 \log$ was not reached in our clinical trial results.

Similar outcome was reported in the only RCT to date that evaluated microbial outcome promoted by PACT in dental caries. Gugliemi et al. [31] showed a range of log reduction from 0.91 to 1.38 using methylene blue dye and a red low-power laser. Although there have been encouraging reports of the use of PACT to inactivate cariogenic bacteria in large in vitro studies, there have been relatively few reports of their use to reach bacterial reduction in dental caries in vivo as confirmed by recent systematic review of the literature [17].

This inferior bacterial log reduction from clinical trials compared to those reported in in vitro and in situ studies characterize the problematic transfer of laboratorial and preclinical results into clinical reality. Our specific target area for disinfection: infected dentin in the bottom of deep caries lesions accessed before restoration placement presents tubules that hidden bacteria making the bacterial reduction a challenge in this specific target area.

Supplementary to this adversity, over-the-counter products commonly used for cariogenic biofilm inihibition as month washes and toothpastes containing bacteriostatic/antibacterial agents have limited access and efficacy in dentin desinfection before resorative procedures.

A basic prerequisite for the effective use of PACT is the uptake and/or binding of the photosensitizer on the bacterial cell wall or plasma membrane. In this approach, dentin substrate plays an important role in PACT effectiveness. Tissue-substrate related factors such as the ability of the photosensitizer to penetrate dentinal tubules to reach the bacteria in appropriate site and the requirement that light should be delivered to the microorganism with appropriated propagation in dentin tissue are considerable obstacles to the clinical use of PACT in caries lesions [32,33]. Dentin represents a unique substrate, in which the dye need to penetrate in demineralized area to reach the disinfection. Previous study [32] evaluated the degree of the penetration of toluidine blue orto (TBO) in artificial caries lesions, in which the rate of TBO penetration was detected up to about $\sim 50 \mu \mathrm{m}$. However, PACT aim to reduce the bacterial core in caries-affected dentin and not reach the tissue sterilization. The degree of penetration of photosensitizer also arise concerns related to biocompatibility of these compounds to pulp tissue. Recent study using demineralized dentin disk showed no indirect harmful effect of PACT to dental pulp cells [13]. 

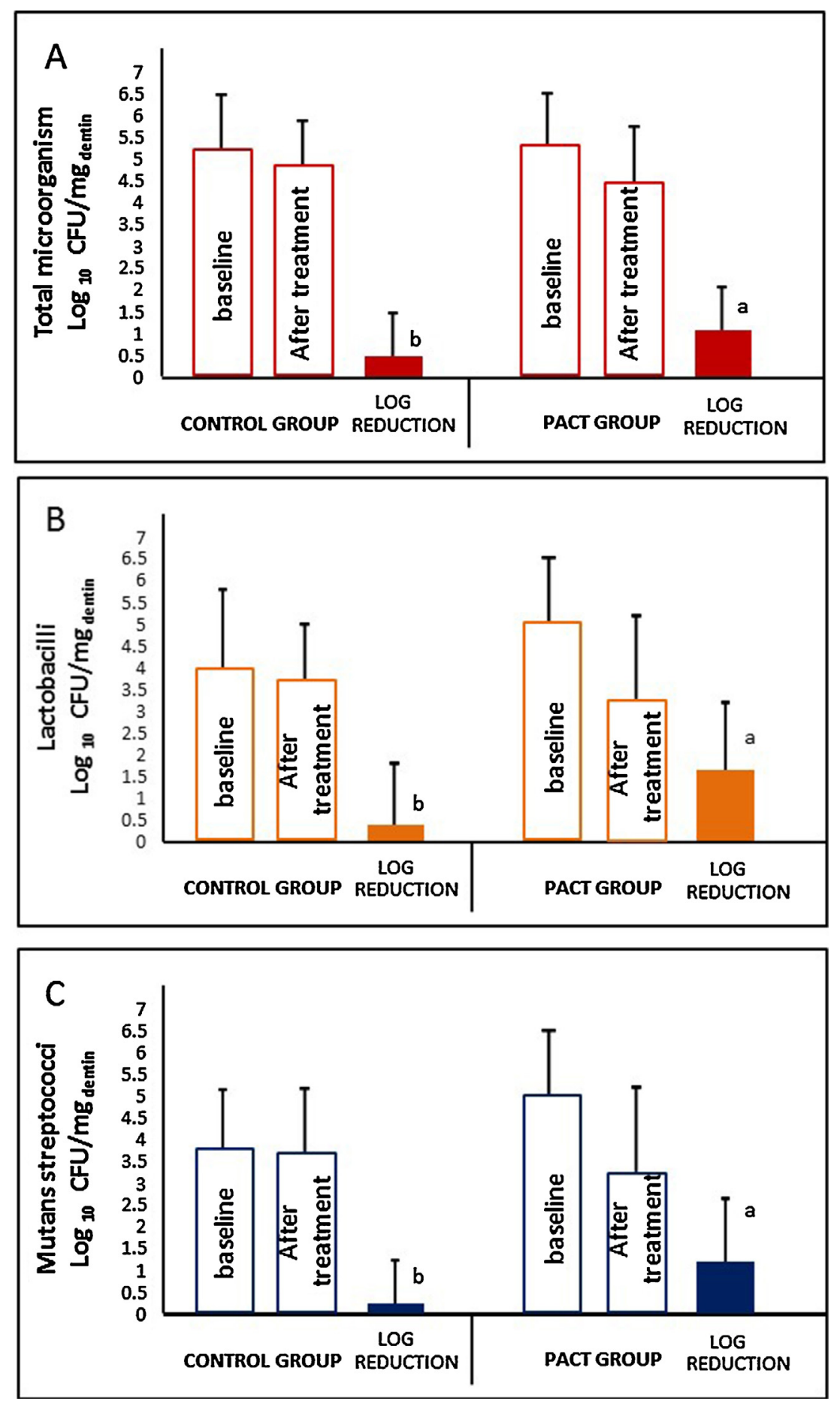

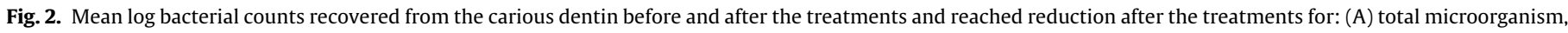
(B) Lactobacilli, and C. S. mutans. Different letters correspond to a significant statistic difference $(p \leq 0.05)$.

In order to attain high antibacterial activity with PACT, alterations in chosen parameters such as irradiation time and the concentration of photosensitizer or the pre-irradiation time can been suggested as strategies to improve PACT performance in vivo. Moreover, previous studies have highlighted the dentin microbiota change under sealing carious lesions/restorations with reduction in the microbiota load and genotypic diversity of the surviving microbiota after long term evaluation [34,35]. The prompt bacterial reduction achieved by PACT in association with these results obtained by stepwise excavation over the time can reach a final pronounced outcome that might jeopardize or even impair no bac- terial viability contributing with the aim of preserving pulp and remaining dentin tissue.

In conclusion, when a new therapy is developed for a condition, initial expectations for clinical outcome are high, though the facing challenges are expected. Overall, the data generated in the current study grades that the concept of PACT may be plausible and it has the promising potential to be a valuable addition to the therapeutic armamentarium for the treatment of deep caries lesions. The evidence supported by available studies so far justifies the research efforts to improve this therapy and PACT is particularly attractive for eliminating bacteria from dentin before restoration. 


\section{Author's contribution}

M.A.S. Melo-Acquisition of data: operator, study coordinator, analysis and interpretation of data, drafting of the manuscript.

J.P.M.L. Rolim-Acquisition of data: microbiological analysis.

V.F. Passos-Acquisition of data: patient screening coordinator.

R.A. Lima-Acquisition of data: patient screening, assistance in clinical procedures.

I.C.J. Zanin-Assistance with study design, critical revision of the manuscript for important intellectual content.

Bruna. M. Codes-Acquisition of data: patient screening, assistance in microbiological procedures.

Suellen.S. Rocha-Acquisition of data: patient screening, assistance in clinical procedures.

L.K.A. Rodrigues-Study concept and design, critical revision of the manuscript for important intellectual content, administrative, technical or material support and Study supervision.

\section{Conflict of interest}

The authors have no pecuniary or other personal interest, direct or indirect, in any matter that raises or may raise a conflict of interests related to this study.

\section{Acknowledgments}

This study was supported by CNPq620160/2006-3. The authors thank Sarah F.F Guedes and Weslanny A. Morais for their help with experimental procedures. We also thank all the volunteers for their valuable contribution.

\section{References}

[1] L. Bjørndal, E.A. Kidd, The treatment of deep dentine caries lesions, Dent. Update 32 (September (7)) (2005), 402-404, 407-410, 413.

[2] D. Ricketts, T. Lamont, N.P. Innes, E. Kidd, J.E. Clarkson, Operative caries management in adults and children cochrane database, Syst. Rev. 28 (March (3)) (2013), http://dx.doi.org/10.1002/14651858.CD003808.pub3, CD003808

[3] F. Schwendicke, H. Meyer-Lueckel, C. Dörfer, S. Paris, Failure of incompletely excavated teeth-a systematic review, J. Dent. 41 (July (7)) (2013) 569-580.

[4] A.I. Orhan, F.T. Oz, K. Orhan, Pulp exposure occurrence and outcomes after 1or 2-visit indirect pulp therapy vs complete caries removal in primary and permanent molars, Pediatr. Dent. 32 (July-August (4)) (2010) 347-355

[5] A.I. Orhan, F.T. Oz, B. Ozcelik, K. Orhan, A clinical and microbiological comparative study of deep carious lesion treatment in deciduous and young permanent molars, Clin. Oral. Investig. 12 (December (4)) (2008) 369-378.

[6] M. Hayashi, M. Fujitani, C. Yamaki, Y. Momoi, Ways of enhancing pulp preservation by stepwise excavation-a systematic review, J. Dent. 39 (February (2)) (2011) 95-107.

[7] M.A. Melo, Photodynamic antimicrobial chemotherapy as a strategy for dental caries: building a more conservative therapy in restorative dentistry, Photomed. Laser Surg. 32 (November (11)) (2014) 589-591.

[8] J.A. Williams, G.J. Pearson, M.J. Colles, Antibacterial action of photoactivated disinfection (PAD) used on endodontic bacteria in planktonic suspension and in artificial and human root canals, J. Dent. Res. 34 (2006) 363-371.

[9] K. Konopka, T. Goslinski, Photodynamic therapy in dentistry, J. Dent. Res. 86 (2007) 694-707.

[10] J.A. Williams, G.J. Pearson, M.J. Colles, M. Wilson, The effect of variable energy input from a novel light source on the photoactivated bactericidal action of toluidine blue O on streptococcus mutans, Caries Res. 37 (2003) 190-193.

[11] I.C.J. Zanin, R.B. Goncalves, A.J. Brugnera Jr, C.K. Hope, J. Pratten, Susceptibility of Streptococcus mutans biofilms to photodynamic therapy: an in vitro study, J. Antimicrob. Chemother. 56 (2005) 324-330.

[12] I.C. Zanin, M.M. Lobo, L.K. Rodrigues, L.A. Pimenta, J.F. Hofling, R.B. Goncalves, Photosensitization of in vitro biofilms by toluidine blue $\mathrm{O}$ combined with a light-emitting diode, Eur. J. Oral. Sci. 114 (2006) 64-69.

[13] I.M. Diniz, I.D. Horta, C.S. Azevedo, T.R. Elmadjian, A.B. Matos, M.R. Simionato, M.M. Marques, Antimicrobial photodynamic therapy: a promise candidate for caries lesions treatment, Photodiagn. Photodyn. Ther. (May (19)) (2015), http://dx.doi.org/10.1016/j.pdpdt.2015.04.006, pii: S1572-1000(15)00041-1 (Epub ahead of print).
[14] M.A.S. Melo, D.M. De-Paula, J.P.M. Lima, F.M.C. Borges, C. Steiner-Oliveira, M. Nobre-Dos-Santos, I.C.J. Zanin, E.B. Barros, L.K.A. Rodrigues, In vitro photodynamic antimicrobial chemotherapy in dentine contaminated by cariogenic bacteria, Laser Phys. 20 (2010) 1-10.

[15] J.S.M. Giusti, L. Santos-Pinto, A.C. Pizzolito, K. Helmerson, E. Carvalho-Filho, C. Kurachi, V.S. Bagnato, Antimicrobial photodynamic action on dentin using a light-emitting diode light source, Photomed. Laser Surg. 26 (2008) 279-285.

[16] J.S. Denne, C. Jennison, Estimating the sample size for a t-test using an internal pilot, Stat. Med. 18 (July (13)) (1999) 1575-1585.

[17] G.C. Santin, D.S. Oliveira, R. Galo, M.C. Borsatto, S.A. Corona, Antimicrobial photodynamic therapy and dental plaque: a systematic review of the literature, Sci. World J. (2014) 824538, http://dx.doi.org/10.1155/2014/ 824538, Epub 14.10.14 Review.

[18] H. Gursoy, C. Ozcakir-Tomruk, J. Tanalp, S. Yilmaz, Photodynamic therapy in dentistry: a literature review, Clin. Oral Investig. 17 (May (4)) (2013) 1113-1125, http://dx.doi.org/10.1007/s00784-012-0845-7, Epub 27.09.12 Review.

[19] M. Hayashi, M. Fujitani, C. Yamaki, Y. Momoi, Ways of enhancing pulp preservation by stepwise excavation-a systematic review, J. Dent. 39 (February (2)) (2011) 95-107.

[20] L. Bjørndal, T. Larsen, Changes in the cultivable flora in deep carious lesions following a stepwise excavation procedure, Caries Res. 34 (November-December (6)) (2000) 502-508.

[21] X.J. Fu, Y. Fang, M. Yao, Antimicrobial photodynamic therapy for methicillin-resistant Staphylococcus aureus infection, Biomed. Res. Int. (2013) 159157.

[22] F. Vatansever, W.C. de Melo, P. Avci, D. Vecchio, M. Sadasivam, A. Gupta, R. Chandran, M. Karimi, N.A. Parizotto, R. Yin, G.P. Tegos, M.R. Hamblin, Antimicrobial strategies centered around reactive oxygen species-bactericidal antibiotics, photodynamic therapy, and beyond, FEMS Microbiol. Rev. (Jun (27)) (2013), http://dx.doi.org/10.1111/1574-6976.12026 (Epub ahead of print).

[23] N. Hakimiha, F. Khoei, A. Bahador, R. Fekrazad, The susceptibility of Streptococcus mutans to antibacterial photodynamic therapy: a comparison of two different photosensitizers and light sources, J. Appl. Oral Sci. 22 (April (2)) (2014) 80-84

[24] N.C. Araújo, C.R. Fontana, V.S. Bagnato, M.E. Gerbi, Photodynamic effects of curcumin against cariogenic pathogens, Photomed. Laser Surg. 30 (July (7)) (2012) 393-399.

[25] Y.H. Lee, H.W. Park, J.H. Lee, H.W. Seo, S.Y. Lee, The photodynamic therapy on Streptococcus mutans biofilms using erythrosine and dental halogen curing unit, Int. J. Oral Sci. 4 (December (4)) (2012) 196-201.

[26] I.M. Bevilacqua, R.A. Nicolau, S. Khouri, A. Brugnera Jr., G.R. Teodoro, R.A. Zângaro, M.T. Pacheco, The impact of photodynamic therapy on the viability of Streptococcus mutans in a planktonic culture, Photomed. Laser Surg. 25 (December (6)) (2007) 513-518.

[27] A.H. Teixeira, E.S. Pereira, L.K. Rodrigues, D. Saxena, S. Duarte, I.C. Zanin, Effect of photodynamic antimicrobial chemotherapy on in vitro and in situ biofilms, Caries Res. 46 (6) (2012) 549-554.

[28] M.A. Monteiro-Oliveira, L.K.A. Rodrigues, M.A.S. Melo, M. Nobre-dos-Santos, Photodynamic therapy effect in carious bovine dentin-an in vitro study, J. Oral Laser Appl. 10 (1) (2010) 29-36.

[29] J.P.M. Lima, M.A. Sampaio De Melo, F.M. Borges, A.H. Teixeira, C. Steiner-Oliveira, M. Nobre Dos Santos, L.K. Rodrigues, I.C. Zanin, Evaluation of the antimicrobial effect of photodynamic antimicrobial therapy in an in situ model of dentine caries, Eur. J. Oral Sci. 117 (2009) 568-574.

[30] F.M.C. Borges, M.A.S. Melo, J.P.M. Lima, I.C.J. Zanin, L.K.A. Rodrigues, M. Nobre-dos-santos, Evaluation of the effect of photodynamic antimicrobial therapy in dentin caries: a pilot in vivo study SPIE. (Org.), SPIE Proceedings 7549 (2010) 75490

[31] C.A. Guglielmi, M.R. Simionato, K.M. Ramalho, J.C.P. Imparato, S.L. Pinheiro, M.A.A.C. Luz, Clinical use of photodynamic antimicrobial chemotherapy for the treatment of deep carious lesions, J. Biomed. Opt. 16 (8) (2011) 1-7, 088003.

[32] M.A. Melo, J.P. Rolim, I.C. Zanin, J.J. Silva, A.R. Paschoal, A.P. Ayala, L.K. Rodrigues, A comparative study of the photosensitizer penetration into artificial caries lesions in dentin measured by the confocal Raman microscopy, Photochem. Photobiol. (October (15)) (2013), http://dx.doi.org/ 10.1111/php.12186 (Epub ahead of print).

[33] J.Y. Nagata, N. Hioka, E. Kimura, V.R. Batistela, R.S. Terada, A.X. Graciano, M.L. Baesso, M.F. Hayacibara, Antibacterial photodynamic therapy for dental caries: evaluation of the photosensitizers used and light source properties, Photodiagn. Photodyn. Ther. 9 (June (2)) (2012) 122-131.

[34] M. Maltz, S.L. Henz, E.F. de Oliveira, J.J. Jardim, Conventional caries removal and sealed caries in permanent teeth: a microbiological evaluation, J. Dent. 40 (September (9)) (2012) 776-782.

[35] J.S. Paddick, S.R. Brailsford, E.A. Kidd, D. Beighton, Phenotypic and genotypic selection of microbiota surviving under dental restorations, Appl. Environ. Microbiol. 71 (May (5)) (2005) 2467-2472. 